\title{
Reliability-based design optimization analysis of a piezoelectric engine
}

\section{Analyse basée optimisation fiabiliste d'un moteur piézo-électrique}

\author{
Bouchaïb Radi ${ }^{1}$, Abdelkhalak El Hami ${ }^{2}$ \\ ${ }^{1}$ LIMII, FST Settat, BP : 577, Route de Casablanca, Settat, Morocco, bouchaib.radi@yahoo.fr \\ ${ }^{2}$ LMN, National Institute of Applied Sciences Rouen, 76801 St Etienne du Rouvray, France, aelhami@insa-rouen.fr
}

\begin{abstract}
In this paper, we study extensions of the RBDO of the piezoelectric engines with travelling wave taking into account the dynamic contact between the different components (stator and rotor). Generally, the life of these engines is limited by important abrasion of the different components. So, the notion of random variables and the risk of failure must be integrated in the mechanical analysis to ensure the good working of the system. The numerical treatment of the dynamic contact inside the motor is presented with the good choice of relaxation factors. The RBDO is often formulated as a minimization of the initial structural cost under constraints imposed on the values of elemental reliability indices corresponding to various limit states. The classical RBDO leads to high computing time and weak convergence, but a hybrid method has been proposed to overcame these two drawbacks. The efficiency of the hybrid method has been demonstrated on static and dynamic cases with extension to the variability of the probabilistic model. We propose two methods: the dynamic hybrid method and the frequencies hybrid method as extension of the improved hybrid method presented in further works.

KEYWORDS. Reliability analysis, Finite element analysis, Reliability-based design Optimization, dynamic contact.
\end{abstract}

\section{Introduction}

Many sources of energy were explored to ensure the excitation of the vibrations of volume on the stator element of the engine. The most significant results were recorded with devices exploiting of the forces of origin electromagnetic or electrodynamic, magnetostrictive or piezoelectric. Japan took over this work since the beginning of the years 1980, with significant means which in particular allowed the design and then the first industrial developments of piezoelectric engines (driving SHINSEI, engines of Zoom of the apparatuses auto focus GUN...) are appeared.

The generation of a progressive wave of volume imposes the respect of geometrical constraints and mechanics relating to the periodicity of the engine's structure. Under normal conditions of operation, the engines are subjected to:

- An axial static loading of pre-stressing actuating of the axial and radial deformations stator and rotor.

- A dynamic excitation of the stator, involving deformations of inflection out-plan which, while being propagated in the volume of the stator, create by drive, a rigid displacement of the rotor's body.

- Efforts of contact and friction static and dynamic to the interface of contact between the stator and the coating of friction.

The aim of this study is to propose a numerical modelling by the finite element method of the mechanical behaviour of piezoelectric engine SHINSEI USR 60 pennies dynamic loading taking into account the contact without friction by using the reliability analysis: the notion of aleas as introduced in our numerical simulation.

Contact problems are treated using an augmented Lagrangian approach to identify the candidate contact surface and contact stresses (Baba et al 2000) and the dynamic treatment is solved using a time integration scheme. The time integration parameters are specially selected to ensure that the solution of 
dynamic contact problem in unconditionally stable and reduce significantly the spurious highfrequency modes, which persist in the traditional Newmark method.

The stochastic role of each parameter of design in the default risk is highlighted. For this reliability analysis of the structure, we propose a coupling direct mechanic-reliability between the augmented Lagrangian method to solve the contact, developed on a computer code by finite elements, and probabilistic method FORM (Moro et al 2002).

By introducing a risks unit with the mechanical problem, we evaluate the probability that the constraints of contact in the coating of friction exceed a value threshold safety and limit the lifespan initially envisaged when designing engine USR 60. Deterministic design optimization enhanced by reliability performances and formulated within the probabilistic framework is called Reliability-based Design Optimization (RBDO). Generally, the purpose of the Reliability-Based Design Optimization (RBDO) is to design structures that should be economic and reliable by introducing safety criteria in the optimization procedure and it is often formulated as a minimization of the initial structural cost under constraints imposed on the values of elemental reliability indices corresponding to various limit states (Madsen and Friis Hansen 1991 ; Kleiber et al. 1999). This method is used to perform new design to the studied engine.

We propose two methods: the dynamic hybrid method and the frequencies hybrid method as extension of the improved hybrid method presented in further works. The discussion of these different approaches based on the RBDO is given. The numerical results show the efficiency of these two proposed methods and the efficiency of each method is done. In this work, we model the Young's modules of three materials and the external loading by the normal law. The results obtained then enable us to propose a whole of recommendations to optimize the reliability of piezoelectric engine USR 60.

\section{Reliability contact analysis}

\subsection{Treatment of the dynamic contact}

Contact between solids is generally governed by two constraints which can be written as follows:

$$
\begin{aligned}
& u_{N}-\delta<0 \Rightarrow r_{N}=0 \\
& u_{N}-\delta=0 \Rightarrow r_{N} \leq 0
\end{aligned}
$$

where $\delta$ represents the gap between the contacting bodies, $u_{N}$ the normal component of the displacement field and $r_{N}$ the normal reaction (Kikuchi and Oden 1988).

From the Hamilton principle, the system energy and work of external forces can be written in the following form:

$$
\begin{array}{r}
\Pi(u)=\int_{t_{1}}^{t_{2}} \int_{V} \frac{1}{2} \varepsilon^{T} D \varepsilon d V d t-\int_{t_{1}}^{t_{2}} \int_{V} \frac{1}{2} \rho\left(\frac{d u}{d t}\right)^{T}\left(\frac{d u}{d t}\right) d V d t \\
-\int_{t_{1}}^{t_{2}} \int_{S_{1}} u^{T} P d S d t-\int_{t_{1}}^{t_{2}} \int_{S_{2}} u^{T} R d S d t-\int_{t_{1}}^{t_{2}} \int_{S_{1}} u^{T} R_{C} d S d t
\end{array}
$$

Enforcement of the zero-penetration condition on contacting boundaries yields:

$T^{T} u-\delta \geq 0, \quad u \in S_{C}$. Where, $\varepsilon$ is the strain vector; $\mathrm{D}$ is the material matrix; $\rho$ is the mass density; $\mathrm{u}$ is the displacement vector; $\mathrm{P}$ is the external load vector; $\mathrm{R}$ is the reaction force vector on prescribed 
displacement boundary; $\mathrm{T}$ is the contact constraint matrix; $\mathrm{S}_{1}$ is the boundary with prescribed external forces; $S_{2}$ is the boundary with prescribed displacements and $S_{c}$ is the contacting boundary.

The augmented Lagrangian approach relative to the dynamic contact problem is given by the weak form of the equilibrium state:

$$
\Pi^{*}(u, \delta u)=\Pi(u, \delta u)+\int_{t_{1}}^{t_{2}} \int_{S_{c}}\left(T^{T} \delta u-\delta\right)^{T} r_{N} d t
$$

where $\delta$ is the gap between two contacting bodies.

This nonlinear equation must be solved but it cannot be solved directly, so $r_{N}$ is considered as known via an iterative process using the Newton-Raphson method (Radi et al 1998). The reaction $r_{N}$ is written in function of a Lagrange multiplier and a penalty coefficient as follows:

$$
r_{N}=\lambda_{n}+\varepsilon_{n} u_{n}
$$

The dynamic treatment is done using the finite difference method in the time domain to establish a relationship between acceleration, velocity and displacement fields. The Newmark method (Newmark 1959), the most frequently used implicit time integration scheme, allows us to approximate velocities and displacements at time $\mathrm{t}+\Delta \mathrm{t}$ as follows:

$$
\begin{gathered}
{ }^{t+\Delta t} \dot{U}={ }^{t} \dot{U}+\left[(1-\gamma)^{t} \ddot{U}+\gamma \quad{ }^{t+\Delta t} \ddot{U}\right] \Delta t, \\
{ }^{t+\Delta t} U={ }^{t} U+\dot{U} \Delta t+\left[\left(\frac{1}{2}-\beta\right)^{t} \ddot{U}+\beta \quad \ddot{U}\right] \Delta t^{2},
\end{gathered}
$$

It has been shown that the use of the trapezoidal rule $(\gamma=0.5$ and $\beta=0.25)$ with a fully implicit treatment of the contact constraints produces oscillations, which can be significant as the time steps and spacial discretization are defined (Chung and Hulbert 1993). So, the equation of motion is modified as follows:

$$
M^{(t+\Delta t)-\alpha_{B}} \ddot{U}+C^{(t+\Delta t)-\alpha_{H}} \dot{U}+K^{(t+\Delta t)-\alpha_{H}} U={ }^{(t+\Delta t)-\alpha_{H}} F,
$$

where

$$
\begin{aligned}
{ }^{(t+\Delta t)-\alpha_{H}} U & =(1-\gamma)^{t+\Delta t} U+\alpha_{H}{ }^{t} U, \\
{ }^{(t+\Delta t)-\alpha_{H}} \dot{U} & =\left(1-\alpha_{H}\right)^{t+\Delta t} \dot{U}+\alpha_{H}{ }^{t} \dot{U}, \\
{ }^{(t+\Delta t)-\alpha_{B}} \ddot{U} & =\left(1-\alpha_{B}\right)^{t+\Delta t} \ddot{U}+\alpha_{B}{ }^{t} \ddot{U}, \\
{ }_{(t+\Delta t)-\alpha_{H}} F & =\left(1-\alpha_{H}\right)^{t+\Delta t} F+\alpha_{H}{ }^{t} F,
\end{aligned}
$$

The Newmark time integration scheme is used to solve the above equation of motion. In this study, we used $\gamma=0.5$ and $\beta=0.25$, with the additional parameters $\alpha_{B}=0.5$ and $\alpha_{H}=0.5$ for the integration constants. Earlier work by the authors indicates that these parameters result in second-order accuracy, unconditional stability, energy and momentum conservation, and not cause numerical dissipation (Czekansk et al 1998). 


\section{Reliability-based design optimization}

It is strong need to integrate the reliability analysis in the optimization process to control the reliability level and to minimize the structural cost or weight in the non-critical regions of the structure. The ultimate goal of design under uncertainty is to reach an optimum in terms of total cost. In principle, an optimum balance between structural system reliability and other conflicting societal goals must be obtained. The integration of reliability analysis into engineering design optimization is termed Reliability-based Design Optimization (RBDO). Traditionally, the solution of the RBDO model is achieved by alternating reliability and optimization iterations. This approach leads to low numerical efficiency, which is disadvantageous for engineering applications on real structures. In order to avoid this difficulty, the hybrid RBDO methods are proposed (Kharmanda et al 2001, Kharmanda et al 2002).

\subsection{Hybrid RBDO method}

To improve the numerical performance, the hybrid approach consists in minimizing a new form of the objective function $F(x, y)$ subject to a limit state and to deterministic and reliability constraints, as:

$\begin{array}{ccc}\min _{x, y} & F(x, y)=f(x) \times d_{\beta}(x, y) \\ \text { subjectto. } G(x, y) & \leq 0 \\ & g_{k}(x, y) & \leq 0 \\ \text { and } & d_{\beta}(x, y) & \geq \beta_{t}\end{array}$

Here, $d_{\beta}(x, y)$ is the distance in the hybrid space between the optimum and the design point, $d_{\beta}(x, y)=d(u)$. The minimization of the function $F(x, y)$ is carried out in the Hybrid Design space (HDS) of deterministic variable $x$ and random variable $y$.

In Kharmanda et al (2002), it is shown that this method reduced the computational time almost $80 \%$ relative to the classical RBDO approach. Using the hybrid one, the optimization process is carried out in the Hybrid Design space, where all numerical information about the optimization process can be modelled. Furthermore, the classical RBDO approach has weak convergence stability because is carried out in two spaces (physical and normalized space).

The efficiency of the hybrid approach allows extension for solving more complex problems. So, we have proposed the dynamic hybrid method (Mohsine et al 2005). The efficiency of this method is showed in (Mohsine et al 2006).

\subsection{Dynamic Hybrid method}

The problems in dynamics of the structures take a very big importance, notably the tendency to construct more and more flexible and subject structures to the excitations that fluctuate more and more quickly in the time. The solution of the above nested problems leads to very large computational time, especially for large-scale structures. The integration of the procedures of optimization based reliability, for the conception of the dynamic systems, gives birth of a new method named dynamic hybrid method. In this new method, we propose the integration of the factor time like a primordial element (Mohsine et al 2005). Besides this method takes advantage of all positive features of the classic hybrid method.

\section{Formulation}

In order to improve the numerical performance, the hybrid approach consists in minimizing a new form of the objective function $F(\mathbf{x}, \mathbf{y})$ subject to a limit state and to deterministic as well as to reliability constraints: 


$$
\begin{aligned}
& \min \quad: f(\mathbf{x}) \cdot d_{\beta}(\mathbf{x}, \mathbf{y}, t) \\
& \text { subject to }: G(\mathbf{x}, \mathbf{y}, t) \leq 0 \\
& : d_{\beta}(\mathbf{x}, \mathbf{y}, t) \leq \beta_{c}(t) \quad \forall t \in\left[\begin{array}{ll}
0 & T
\end{array}\right] \\
& \text { and } \quad: g_{k}(\mathbf{x}, t) \leq 0
\end{aligned}
$$

In the case $t=0$ (static problem), $d_{\beta}(\mathbf{x}, \mathbf{y})$ is the distance in the hybrid space between the optimum and the design point, $d_{\beta}(\mathbf{x}, \mathbf{y})=d(\mathbf{u})$. The minimization of the function $F(\mathbf{x}, \mathbf{y})$ is carried out in the Hybrid Design Space (HDS) of deterministic variables $\mathbf{x}$ and random variables $\mathbf{y}$.

An example of this HDS is given in figure 1, containing design and random variables, where the reliability levels $d_{\beta}$ can be represented by ellipses in case of normal distribution, the objective function levels are given by solid curves and the limit state function is represented by dashed level lines except for $G(\mathbf{x}, \mathbf{y})=0$. We can see two important points: the optimal solution $P_{x}^{*}$ and the reliability solution $P_{y}^{*}$ (i.e. the design point found on the curves $G(\mathbf{x}, \mathbf{y})=0$ and $d_{\beta}=\beta_{t}$ ).

\section{Optimality conditions}

The optimization problem under constraint can be reduce to an minimisation problem without constraint by using a hybrid Lagrangian as follows:

$$
L_{H}(x, y, \lambda)=f(x) \cdot d_{\beta}(x, y)+\lambda_{\beta}\left[\beta_{c}-d_{\beta}(x, y)\right]+\lambda_{G} G(x, y, t)+\sum_{k} \lambda_{k} g(x, t)
$$

where $\lambda_{\beta}, \lambda_{\mathrm{G}}$ and $\lambda_{\mathrm{k}}$ are respectively the Lagrange multipliers. The optimality conditions are:

$$
\begin{gathered}
\frac{\partial L_{H}}{\partial x_{i}}=d_{\beta}(x, y) \frac{\partial f}{\partial x_{i}}+\left[f(x)-\lambda_{\beta}\right] \frac{\partial d_{\beta}}{\partial x_{i}}+\lambda_{G} \frac{\partial G}{\partial x_{i}}+\sum_{k} \lambda_{k} \frac{\partial g_{k}}{\partial x_{i}}=0 \\
\frac{\partial L_{H}}{\partial y_{i}}=\left[f(x)-\lambda_{\beta}\right] \frac{\partial d_{\beta}}{\partial y_{i}}+\lambda_{G} \frac{\partial G}{\partial y_{i}}=0 \\
\frac{\partial L_{H}}{\partial \lambda_{\beta}}=\beta_{c}-d_{\beta}(x, y)=0 \text { while } \lambda_{\beta}=0 \\
\frac{\partial L_{H}}{\partial \lambda_{G}}=G(x, y)=0 \\
\frac{\partial L_{H}}{\partial \lambda_{k}}=g_{k}(x)=0 \text { while } \lambda_{k}=0
\end{gathered}
$$




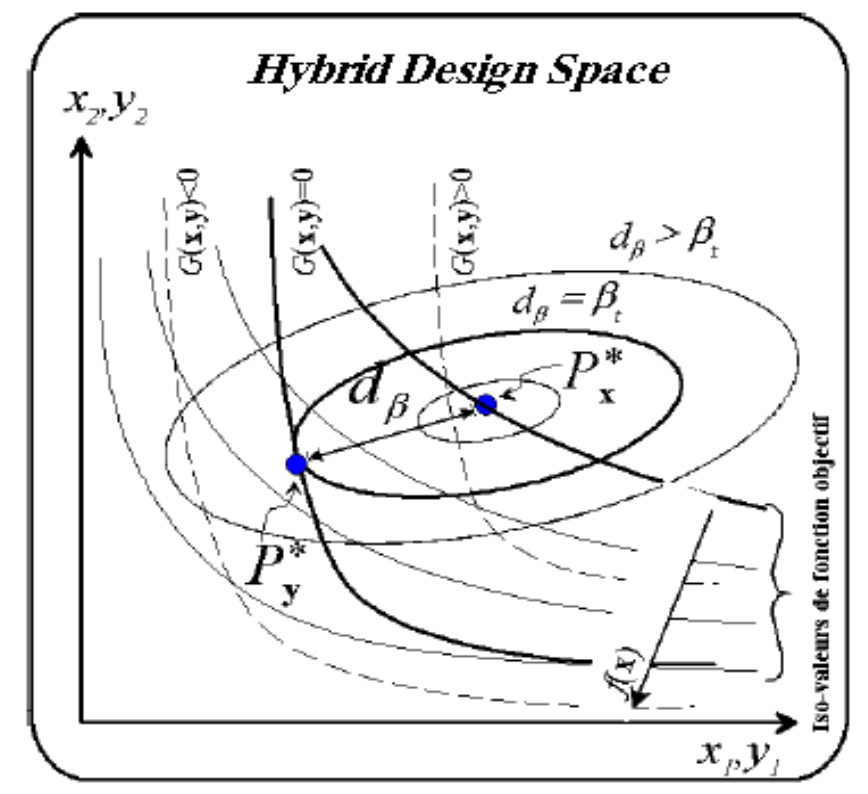

Figure 1. Hybrid Design Space in the case $t=0$

\subsection{Frequencies Hybrid Method (FHM)}

The response of a structure to a dynamic excitation depends, to a large extent, on the first few natural frequencies of the structure. Excessive vibration occurs when the frequency of the dynamic excitation is close to one of the natural frequencies of the structure. In designing most structures, it is often necessary to restrict the fundamental frequency or several of the lower frequencies of the structure to a prescribed range in order to avoid severe vibration. The hybrid formulation will not be able in its traditional formulation to determine the critical region about eigen-frequency.

A new formulation was developed within the framework of calculations into dynamic excitation. The goal of this development is to seek the dangerous frequencies bands relative to different eigenfrequencies. The principal idea is to seek more than one point of design, two design's points per example. These two design's points are used for each iteration to find the lower limit and the upper limit of the frequencies band critical. This critical band coincides with the crucial frequencies band to the neighbourhood of the frequency of the resonance of the structure to optimize (see figure 2). These two boundary-marks are to find to every iteration of the optimization calculation.

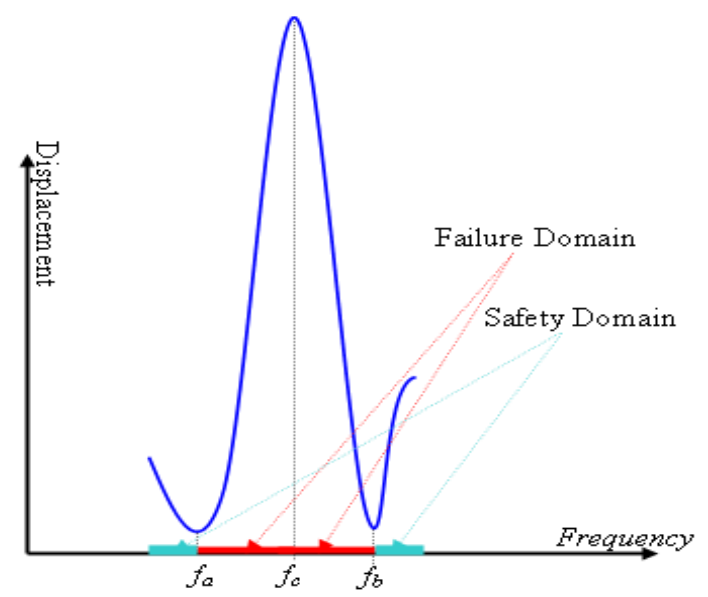

Figure 2. Displacement and eigen-frequency 


\section{Formulation}

The new formulation of frequencies problem:

$$
\begin{aligned}
& \min _{x, y}: F(\mathbf{x}, \mathbf{y})=f(\mathbf{x} .) \cdot d_{\beta_{1}}(\mathbf{x}, \mathbf{y}) \cdot d_{\beta_{2}}(\mathbf{x}, \mathbf{y}) \\
& \text { subject to }: G(\mathbf{x}, \mathbf{y}) \leq 0 \\
& : g_{k}(\mathbf{x}) \leq 0 \\
& \text { and } \quad: d_{\beta_{1}}(\mathbf{x}, \mathbf{y}) \geq \beta_{t} \\
& : d_{\beta_{2}}(\mathbf{x}, \mathbf{y}) \geq \beta_{t}
\end{aligned}
$$

An example of this Hybrid Design Space and Displacement and eigen-frequency is given in the figure 3, in this case, we can see three important points: the optimal solution $P_{x}^{*}$ and two point of the reliability solution $P_{y}^{*}$ (i.e. the design point found on the curves $G(\mathbf{x}, \mathbf{y})=0$ and $d_{\beta}=\beta_{t}$ ). All information about the RBDO problem can be found in this space.

Optimality conditions:

For this problem, the Langrangian is written as:

$$
\begin{aligned}
& \left.\left.L_{H}(x, y, \lambda)=f(x) \cdot d_{\beta_{a}}(x, y) \cdot d_{\beta_{b}}(x, y)+\lambda_{\beta_{a}} \mid \beta_{t}-d_{\beta_{a}}(x, y)\right\rfloor+\lambda_{\beta_{b}} \mid \beta_{t}-d_{\beta_{b}}(x, y)\right\rfloor \\
& +\lambda_{G} G(x, y)+\sum_{k} \lambda_{k} g(x)
\end{aligned}
$$

The optimality conditions of this Lagrangian are:

$$
\begin{gathered}
\frac{\partial L_{H}}{\partial x_{i}}=d_{\beta_{a}}(x, y) d_{\beta_{b}}(x, y) \frac{\partial f}{\partial x_{i}}+\left[f(x) d_{\beta_{b}}(x, y)-\lambda_{\beta_{a}}\right] \frac{\partial d_{\beta_{a}}}{\partial x_{i}} \\
+\left[f(x) d_{\beta_{a}}(x, y)-\lambda_{\beta_{b}}\right] \frac{\partial d_{\beta_{b}}}{\partial x_{i}}+\lambda_{G} \frac{\partial G}{\partial x_{i}}+\sum_{k} \lambda_{k} \frac{\partial g_{k}}{\partial x_{i}}=0 \\
\frac{\partial L_{H}}{\partial y_{i}}=\left[f(x) d_{\beta_{b}}(x, y)-\lambda_{\beta_{a}}\right] \frac{\partial d_{\beta_{a}}}{\partial y_{i}}+\left[f(x) d_{\beta_{a}}(x, y)-\lambda_{\beta_{b}}\right] \frac{\partial d_{\beta_{b}}}{\partial y_{i}}+\lambda_{G} \frac{\partial G}{\partial y_{i}}=0 \\
\frac{\partial L_{H}}{\partial \lambda_{\beta_{a}}}=\beta_{t}-d_{\beta_{a}}(x, y)=0 \\
\frac{\partial L_{H}}{\partial \lambda_{\beta_{b}}}=\beta_{t}-d_{\beta_{b}}(x, y)=0 \\
\frac{\partial L_{H}}{\partial \lambda_{G}}=G(x, y)=0 \\
\frac{\partial L_{H}}{\partial \lambda_{k}}=g_{k}(x)=0
\end{gathered}
$$

\section{Numerical Results}

In this example, we present a study on the Reliability design optimisation on the stator and the rotor of a piezoelectric engine with annular progressive wave SHINSEI USR 60 (figure 3), which will be subjected to the constraint stress (Le Moal and Cusin 1999). 


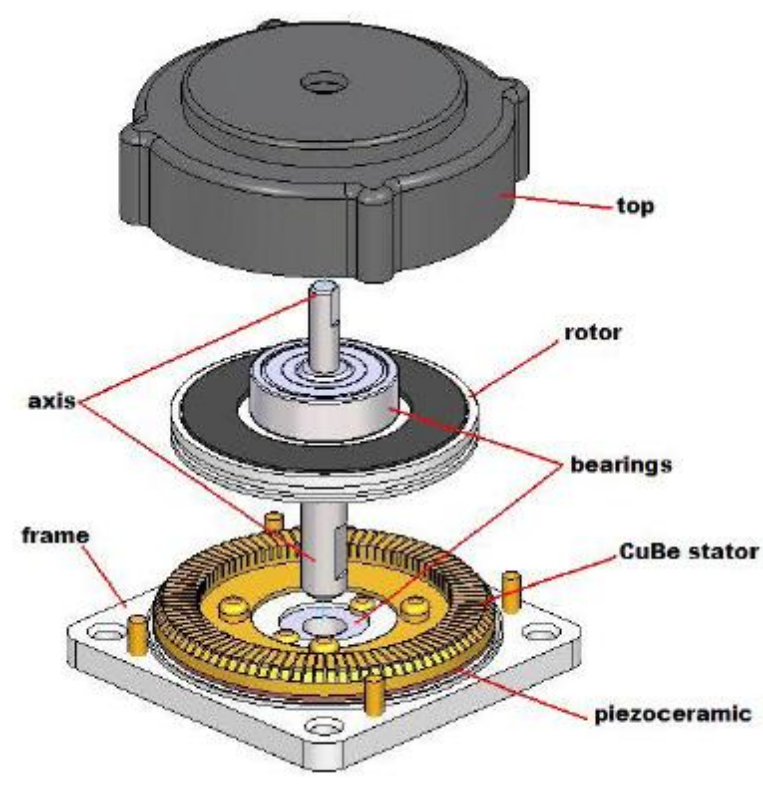

Figure 3. Motor USR 60

\subsection{Geometry and operation}

The operation principle is described on figure 3. This motor is made of two main parts:

- The stator: It is a beryllium-coppper annular plate. At his circumference, teeth are machined to amplify the vibration movement and eliminate the wear particles. At is bottom surface; piezoelectric ceramics are glued to excite the metallic part. The stator is fixed to the frame at its centre. To guarantee the free vibration of the stator ring, a decoupling fold is machined between the centre and the circumference.

- The rotor, it can be separated in 3 zones: the axis, output of the motor; the track friction in contact with the stator and the spring fold linking axis to track and giving the elasticity needed to apply rotor on stator.

Other parts are the frame, the top and bearings. The piezoceramic ring is metallized on one face. The cube stator constitutes the ground electrode. Metallization and polirization are designed to excite a particulate bending mode (depending of the motor's size). This frequency is generally between 30 and $100 \mathrm{Khz}$, thus the name of ultrasonic motor (USM). Excitation at a natural frequency creates a travelling wave; each point of the top surface of the stator has an elliptic motion. The stator is in permanence in contact with the maximum of the wave which has all the same elementary movement. All these elementary displacements drive the rotor by friction. To have good contact conditions, a thin polymer layer can be reported on the rotor or the stator.

The geometrical cross section of the stator and the rotor shows in figures 6 and 7. The body force $F_{\text {ext }}$ on the SHINSEI USR 60 engine, is transmit to the rotor circumferential and punctually done at the ratio $R=21.10^{-3} \mathrm{~m}$ with the value $140 \mathrm{~N}$. The mechanical characteristics of different material and geometry are given in tables 1 and 2 . 

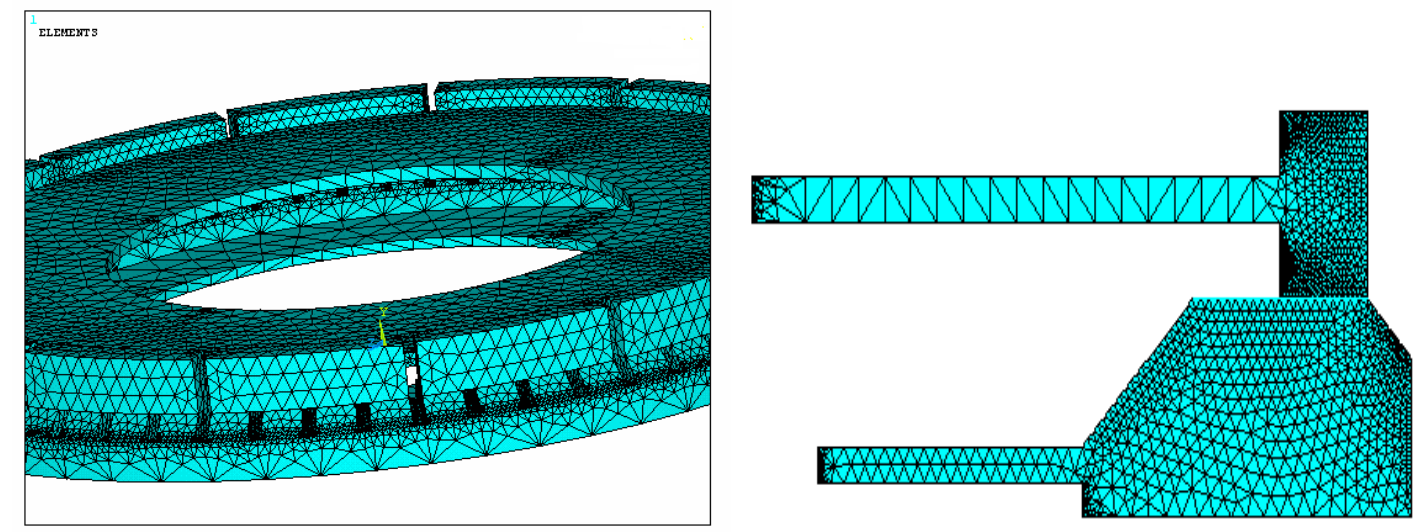

Figure 4. Finite elements modelisation of stator and rotor

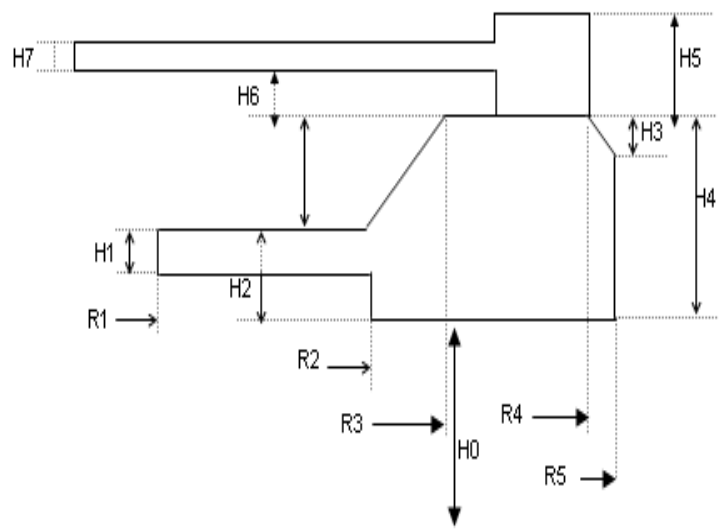

Figure 5. Dimensional parameters of the stator and the rotor

\begin{tabular}{|l|l|l|l|l|l|l|l|l|l|l|l|l|l|}
\hline Parameters & H0 & H1 & H2 & H3 & H4 & H5 & H6 & H7 & R1 & R2 & R3 & R4 & R5 \\
\hline$(\mathrm{mm})$ & 0.5 & 0.6 & 1.2 & 1 & 4.2 & 3.2 & 1.3 & 2.1 & 16.5 & 22.5 & 25 & 29 & 30 \\
\hline
\end{tabular}

Table 1. Geometry characteristics

\begin{tabular}{|c|c|c|c|}
\hline \multicolumn{2}{|c|}{ Rotor } & \multicolumn{2}{c|}{ Stator } \\
\hline $\mathrm{E}(M P a)$ & $\rho\left(K g / m^{3}\right)$ & $\mathrm{E}(M P a)$ & $\begin{array}{c}\rho \\
\left(K g / m^{3}\right)\end{array}$ \\
\hline 27000 & 2700 & 123000 & 8250 \\
\hline
\end{tabular}

Table 2. Mechanical characteristics

\subsection{Results and discussion}

The objective of this study is to show the efficiency and the robustness of the dynamic hybrid method and Frequencies hybrid method. We minimize the cost of structure subject to the stress constraint, the target reliability index constraint and the second part subject to eigen-frequency constraint the target reliability index constraint. The system must satisfy the predefined target 
reliability. The study of the static contact between the rotor and the stator using reliability analysis is done in (El Hami and Moro 2003).

In this numerical test, we want to look about the model reduction by substructures method and the result of the FHM using this model reduction. The complete structure has 13122 active degrees of freedom but the structure's condensed has only 2000 active degrees of freedom. Thus can show the good importance of the use of the substructures method. We take $f_{c}=39 \mathrm{KHz}$ and $\beta_{t}=3.8$, where $r 2, r 3, r 4, h 1, h 2, h 3$ and $h 4$ are grouped in the random vector $\mathbf{y}$ but to optimize the design, the means $m_{r 2}$, $m_{r 3}, m_{r 4}, m_{h 1}, m_{h 2}, m_{h 3}$, and $m_{h 4}$ are grouped in the deterministic vector $\mathbf{x}$, and their standard-deviation equals to $0.1 m_{x}$. The finding results are given in table 5:

\begin{tabular}{|c|c|c|c|c|c|c|}
\hline \multirow{2}{*}{ Variables } & \multicolumn{3}{|c|}{ Complete structure } & \multicolumn{3}{c|}{ Reduced structure } \\
\cline { 2 - 7 } & $\begin{array}{c}\text { Design } \\
\text { point(a) }\end{array}$ & $\begin{array}{c}\text { Optimal } \\
\text { solution }\end{array}$ & $\begin{array}{c}\text { Design point } \\
\text { (b) }\end{array}$ & $\begin{array}{c}\text { Design point } \\
\text { (a) }\end{array}$ & $\begin{array}{c}\text { Optimal } \\
\text { solution }\end{array}$ & $\begin{array}{c}\text { Design point } \\
\text { (b) }\end{array}$ \\
\hline R2 & 22.211 & 21.214 & 20.76 & 22.3 & 21.213 & 20.76 \\
\hline R3 & 25.59 & 23.959 & 25.4 & 25.49 & 23.958 & 25.43 \\
\hline R4 & 29.66 & 27.925 & 28.33 & 29.67 & 27.92 & 28.32 \\
\hline H1 & 0.428 & 0.562 & 0.678 & 0.429 & 0.56 & 0.68 \\
\hline H2 & 1.345 & 1.483 & 1.549 & 1.346 & 1.481 & 1.55 \\
\hline H3 & 1.5 & 1.318 & 1.66 & 1.45 & 1.32 & 1.67 \\
\hline H4 & 3.66 & 3.435 & 3.489 & 3.65 & 3.43 & 3.49 \\
\hline$\beta$ & 3.65 & ------ & 3.6 & 3.62 & ------- & 3.6 \\
\hline $\begin{array}{c}\text { Frequency } \\
{[\mathrm{KHz}]}\end{array}$ & 37.2 & 39.5 & 41 & 37 & 39 & 40.7 \\
\hline $\begin{array}{c}\text { Volume } \\
{\left[\mathrm{mm}{ }^{3}\right]}\end{array}$ & ------- & $42.76 \times 10^{2}$ & -------- & ------- & $42.67 \times 10^{2}$ & -------- \\
\hline
\end{tabular}

Table 3. Results of the FHM with the complete and the reduced structure

The use of the technic based substructures method has reduced substantially the computational time and the active degrees of freedom. The frequencies band is the same for the two approaches. Now, we look about improved frequencies hybrid methods that take this following form:

$$
\begin{array}{cc}
\min _{x, y}: F(x, y)=V(x) \cdot d_{\beta_{1}}(x, y) \cdot d_{\beta_{2}}(x, y) \cdot V\left(m_{y}\right) \\
\text { under: } & f-f_{c}=0 \\
\text { and }: & d_{\beta_{1}}(x, y) \geq \beta_{c} \\
& d_{\beta_{2}}(x, y) \geq \beta_{c}
\end{array}
$$

with the same $f_{c}$ and $\beta_{c}$. Table 4 gives the finding results using this improved FHM:

\begin{tabular}{|c|c|c|c|c|c|c|}
\hline \multirow{2}{*}{ Variables } & \multicolumn{3}{|c|}{ Complete structure } & \multicolumn{3}{c|}{ Reduced structure } \\
\cline { 2 - 7 } & $\begin{array}{c}\text { Conception } \\
\text { Point (a) }\end{array}$ & $\begin{array}{c}\text { Optimal } \\
\text { solution }\end{array}$ & $\begin{array}{c}\text { Conception } \\
\text { point (b) }\end{array}$ & $\begin{array}{c}\text { Conception } \\
\text { point (a) }\end{array}$ & $\begin{array}{c}\text { Optimal } \\
\text { solution }\end{array}$ & $\begin{array}{c}\text { Conception } \\
\text { point (b) }\end{array}$ \\
\hline R2 & 22.410 & 21.968 & 20.067 & 24.37 & 21.959 & 20.035 \\
\hline R3 & 25.995 & 23.730 & 25.824 & 25.781 & 23.676 & 25.908 \\
\hline R4 & 29.546 & 27.016 & 28.207 & 29.863 & 27.098 & 28.116 \\
\hline H1 & 0.40235 & 0.54574 & 0.63311 & 0.40297 & 0.54475 & 0.62517 \\
\hline H2 & 1.3342 & 1.4343 & 1.5457 & 1.3092 & 1.4354 & 1.5081 \\
\hline H3 & 1.4979 & 1.3011 & 1.6633 & 3.6232 & 1.3075 & 3.4829 \\
\hline H4 & 3.6308 & 3.4215 & 3.4779 & 1.5011 & 3.4076 & 1.6850 \\
\hline$\beta$ & 3.6 & ------ & 3.6 & 3.6 & ------- & 3.6 \\
\hline $\begin{array}{c}\text { Frequency } \\
{[\text { KHz] }}\end{array}$ & 37 & 39.5 & 41 & 37 & 39.3 & 41.2 \\
\hline $\begin{array}{c}\text { Volume } \\
{\left[\mathrm{mm}{ }^{3}\right]}\end{array}$ & -------- & $40.24 \times 10^{2}$ & -------- & -------- & $40.20 \times 10^{2}$ & -------- \\
\hline
\end{tabular}


These results show that the desired constraints are satisfied, Moreover the improved FHM reduces the volume of $5.7 \%$ relative to the optimum volume calculated by the FHM. For more details, one can see (Mohsine 2006).

\section{Conclusion}

The hybrid method RBDO includes in its architecture the deterministic and random parameters in only one hybrid space. This space leads on the good control of all parameters of the problem. The time-variant reliability-based optimization is very interesting to make design for an effective cost, durability and lifetime management of engineering system; it's the Dynamic Hybrid Method (DHM). These advantages motivate us, in order to improve this method, to search the failure critical region about eigen-frequency; it's the Frequencies Hybrid Method (FHM). The second numerical test shows the possibility to analyse a reduced model using the RBDO method. Thus reduces substantially the total number of the degrees of freedom. An improved FHM is proposed. This method reduces the optimum volume and the numerical result shows that the substructures technique can be efficient so This improved method makes a good couple between cost, safety and design.

\section{References}

Baba OA, Radi B, Gelin J-C (2000) An Augmented Lagrangian Treatment of the metal forming process, Mathematical and Computer Modelling, vol. 32, issue 10, 171-179, 2000.

Moro T, El Hami A, El Moudni A (2002) Reliability analysis of a mechanical contact between deformable solids, Probabilistic Engineering Mechanics, vol. 17, Issue 3, 227-232.

Kikuchi N and Oden JT (1988) Contact problems in elasticity: a study of variational inequalities and finite element methods, SIAM studies in applied mathematics (8), SIAM, Philadelphia.

Rajashekhar MR and Ellingwood BR (1993) A new look at the response surface approach for reliability analysis, Struct. Safety, 12, 205-220.

Czekansk S, Meguid A, El Abbasi N (1998) On the selection of a second order accurate integration scheme for elastodynamic contact problems, Special Technical Publications of Engineering Mechanics and Design Laboratory, University of Toronto, vol. STP 168/98-1, 1-28.

Kharmanda G, Mohamed A, Lemaire M (2001) New hybrid formulation for reliability-based optimization of structures, The Fourth World Congress of Structural and Multidisciplinary Optimization, WCSMO-4, Dalian, China, June 4-8.

Kharmanda G, Mohamed A, Lemaire M (2002) Efficient reliability-based design optimization using a hybrid space with applications to finite element analysis, Struct. Multidisc. Optim. vol. 24, 233-245.

Mohsine A, Kharmanda G, El Hami A (2005) Reliability-based design optimisation study using normal and lognormal distributions with applications to dynamic structures, 9th International Conference on Structural Safety and Reliability, ICOSSAR05. Rome, Italy, June 19-23.

Mohsine A, Kharmanda G, El Hami A (2006) Improved hybrid method as a robust tool for reliability-based design optimization, Structural and Multidisciplinary Optimization, 32(2), 203-213.

Le Moal P, Cusin P (1999) Optimization of a traveling wave ultrasonic motors using a three dimensional analysis of the contact mechanism at the stator-rotor interface, European Journal of Mechanics A, Solids, vol. 18, p. 1061-1084.

Chung J., Hulbert GM (1993) A time integration algorithm for structural dynamics with improved numerical dissipation: the generalized-method. Journal of Applied Mechanics; 60, 371-375.

Newmark N.M (1959) A method of computation of structural dynamics. Proceedings of the ASCE, Journal of Engineering Mechanics; 8, 67-94.

Madsen HO, Friis Hansen P (1991) Comparison of some algorithms for reliability-based structural optimization and sensitivity analysis, In Brebbia CA, Orszag SA (eds) Reliability and optimization of structural systems. Springer, Berlin-Heidelberg New-York, 443-451. 
Kleiber M, Siemaszko A, Stocki R (1999) Interactive stability-oriented reliability-based design optimization. Computer Methods Appl. Mech. Eng. 168: 243-253.

Radi B, Baba OA, Gelin J-C (1998) Treatment of the frictional contact via a Lagrangian formulation. Mathematical and Computer Mod. 28(4-8): 407-412.

Mohsine A (2006) Contribution à l'optimisation fiabiliste en dynamique des structures mécaniques. PhD Thesis, LMR, INSA de Rouen.

El Hami A, Moro T (2003) Une analyse par la fiabilité des structures des problèmes d'usure du couple stator/rotor dans un moteur piézo-électrique à onde progressive, Revue de la Sûreté de Fonctionnement, 24(2), 21-34. 